\title{
Desyndicausation et ruptures de contrats de communication
}

Bernard Ibal

\section{(2) OpenEdition}

1 Journals

Édition électronique

URL : http://journals.openedition.org/communicationorganisation/1821

DOI : 10.4000/communicationorganisation. 1821

ISSN : $1775-3546$

Éditeur

Presses universitaires de Bordeaux

Édition imprimée

Date de publication : 1 novembre 1995

ISSN : 1168-5549

\section{Référence électronique}

Bernard Ibal, «Desyndicausation et ruptures de contrats de communication », Communication et organisation [En ligne], 8| 1995, mis en ligne le 26 mars 2012, consulté le 30 avril 2019. URL : http:// journals.openedition.org/communicationorganisation/1821 ; DOI : 10.4000/ communicationorganisation. 1821

Ce document a été généré automatiquement le 30 avril 2019.

(c) Presses universitaires de Bordeaux 


\title{
Desyndicausation et ruptures de contrats de communication
}

\author{
Bernard Ibal
}

1 Avant de nous justifier sur le fond, nous ne pouvons pas passer sous silence les difficultés formelles d'une telle problématique. La désyndicalisation n'est pas directement un concept des sciences de la communication. Par ailleurs Rodolphe Ghiglione ne semble définir son concept de contrat de communication que dans le cadre de l'interlocution inter-individuelle, alors que le débat social relèverait plutôt de la communication de masse. Enfin comment conjuguer rationnellement les problématiques psychologique et politicologique de Ghiglione et de Sfez même si tous deux tentent de comprendre ce qu'est communiquer.

2 Pour ce dernier point, la rencontre des deux spécialistes de la communication se fait selon nous sur une inversion: pour Ghilglione « communiquer c'est co-construire une réalité à l'aide de systèmes de signes "; pour Sfez le tautisme s'avère le fait d'un système de signes fermé sur lui-même qui préconstruit une réalité, laquelle s'impose aux prétendus locuteurs. Inversion, mais sans doute pas contradiction.

Quant à la communication inter-individuelle, à laquelle s'appliquerait exclusivement l'idée de «contrat de communication » de Ghiglione, il faut dire d'une part que l'action syndicale est presque toujours une communication interlocutive duelle (avec tel salarié ou tel patron ou tel politique) et que d'autre part les exemples de Ghilglione mettent en scène des intra-locuteurs eux-mêmes représentatifs par leur culture d'une population implicite voire explicite ${ }^{1}$. De plus Ghiglione ne néglige pas la communication de masse dans sa problématique des contrats de communication puisqu'il prend pour exemple des publicités ${ }^{2}$.

\section{Un fait : la désyndicalisation française}

Dans « La revue de L'IRES » publiée par l'Institut de Recherches du même nom, cogéré par les six centrales syndicales représentatives françaises, Dominique LABBÉ ${ }^{3}$, écrit cette prophétie sans doute surprenante dans un tel ouvrage: «au rythme actuel, le 
syndicalisme français confédéré aura quasiment disparu des entreprises françaises dans moins d'un quart de siècle» (numéro 16 automne 94 p.94/95). Quelle est la chronique de cette mort annoncée ? Dominique LABBÉ publie une courbe, issue d'études concordantes, qui montre que le taux de syndicalisation ${ }^{4}$ est passé en France entre 1949 et 1991 de 50 \% à $10 \%$ avec un relatif étiage de 25 à $30 \%$ de 1958 à 1981.

5 Le phénomène est mondial même si son paroxysme en Occident est d'abord français. Jelle Wisser de l'Université d'Amsterdam ${ }^{5}$ rappelle que le taux de syndicalisation aux USA est passé de $23 \%$ à $16 \%$ de 1980 à 1989, et celui du Japon de $30 \%$ à $25 \%$ durant la même période. En Europe, la chute pourrait paraitre moindre selon la même source et durant la même décennie: de $41 \%$ à $34 \%$. Mais cette dernière statistique comprend les pays Scandinaves dont la forte progression jusqu'en 1988 fait exception, et ne comprend ni le Portugal ni la Grèce, ni l'Espagne qui est en forte baisse. A s'en tenir aux pays de la C.E.E de 1989 le taux de syndicalisation européen serait passé de 38,1 \% à 30,1 \% de 1980 à 1989.

Notons l'hétérogénéité de la désyndicalisation européenne : très forte baisse en Espagne, France, Grande Bretagne et Pays Bas. Baisse très légère en Belgique, Luxembourg, RFA, Autriche et Danemark. Par ailleurs les comparaisons sont très difficiles et aléatoires : fiabilité douteuse des données (en Espagne par exemple), contenu différent de ces données (mélange ou non, par exemple, des actifs et des retraités), et surtout différence de rôles des syndicats qui dans de nombreux pays comme la Belgique, ont par exemple la responsabilité de distribuer l'assurance-chômage et même les primes de fin d'année. En outre la très forte chute des pays cités masque de grandes disparités : la Grande Bretagne est encore à 33,5\% en 1989 tandis que l'Espagne et la France qui sont respectivement autour de $12 \%$ et $10 \%$, sont les lanternes rouges. A l'opposé au sein de la C.E.E le Danemark est encore à 74,4 \% en 1989 malgré sa légère baisse.

7 En revanche existe un facteur d'homogénéité européen de la désyndicalisation : le divorce grandissant entre le profil socio-professionnel de la majorité des syndiqués et le profil socio-professionnel (changeant) de la majorité de l'ensemble des salariés. Ce que Adelheid HEGE dit de l'Allemagne ${ }^{6}$ pourrait, à quelques variantes numériques près, être dit de toute la Communauté Européenne et de la France en particulier: «Le secteur des services occupe aujourd'hui $57 \%$ de l'emploi global. Par contre, le taux de syndicalisation y plafonne à $20 \%$, soit un salarié sur 5 au total. Le syndicalisme allemand est un syndicalisme très largement ouvrier et masculin. Les $2 / 3$ des syndiqués sont des ouvriers et les $3 / 4$ sont des hommes. La place occupée par les femmes est restreinte, et celle des jeunes encore plus puisqu'ils ne représentent que $14 \%$ des effectifs syndiqués ». Le dirigeant de l'IG Métal confirme cette fracture en parlant de sa propre organisation : « Les ouvriers de la métallurgie adhèrent à IG Métal pour $75 \%$ d'entre eux, ce pourcentage tombe à $22 \%$ dans la population d'employés de la métallurgie.» Bref les nouvelles catégories de salariés sont désyndicalisées..

8 C'est ainsi que faute de statistiques syndicales assez fiables, on estime ${ }^{7}$ que le secteur public au sens large occupe en France environ $25 \%$ des salariés, et rassemble pourtant $50 \%$ de l'ensemble des syndiqués. La CGT a précisé 8 que le secteur privé ne concerne que $46,3 \%$ de ses syndiqués et que la CGT est «un peu vieillissante». Ajoutons que ce syndicat, même dans le privé, est surtout implanté dans les grandes entreprises, alors que l'économie et l'emploi se développent surtout dans les P.M.E quasi désertes de syndicats. 
Dominique LABBÉ parle lui aussi de divorce entre les syndicats et les salariés, mais d'un divorce curieusement encore incompris : « il s'agit d'un véritable divorce dont les causes n'ont jamais été clairement expliquées » (idem supra p. 88).

\section{Une méthode d'analyse : Les contrats de communication de R. Ghiglione ${ }^{9}$}

Dans la mouvance qualifiée de gauche, aussi bien chez les syndicalistes que chez les analystes, les causes de la désyndicalisation étaient imputées principalement au chômage, à la désinflation et à son corollaire la stagnation des salaires, à l'individualisme des salariés et à la mauvaise volonté patronale ${ }^{10}$. Dans une mouvance qualifiée de droite, on parlait plutôt de syndicats trop généralistes, pas assez corporatistes et catégoriels, et surtout trop politisés et divisés. Tout le monde peut ici avoir raison ensemble, d'autant plus que Dominique LABBE qui dans son article de 1994 insiste vivement sur le manque de corporatisme de terrain et sur l'indigestion de politique, reconnaît que ses conclusions "sont assez éloignées des thèses habituellement reçues sur le syndicalisme français (celles de chômage, d'individualisme, etc.), thèses que nous partagions au début de nos recherches ». Peut-on espérer trouver une étiologie cohérente plutôt qu'un catalogue de raisons de la désyndicalisation?

11 Puisque tout le monde reconnaît le divorce entre les syndicats et les syndicables, il faut bien partir de cette évidence: ça ne passe plus, ça ne communique plus entre les syndicats et les salariés. Et ça ressemble beaucoup à une rupture du «contrat de communication »

\section{Rupture du « contrat de communication » entre les syndicats et les salariés}

Entre syndicats et salariés semble s'instaurer ce que Ghiglione appelle une "situation potentiellement communicative ». Il y a un enjeu: la défense des intérêts moraux et matériels des salariés. Il y a une pertinence reconnue : face au patronat et à l'État la seule solution est la solidarité des salariés. Il y a réciprocité acceptée : les syndicats anticipent et organisent les solidarités dans leur démocratie interne et dans leur pluralisme; les salariés par leurs adhésions et leurs choix militants donnent force et consistance à cette solidarité. D’ailleurs malgré la désyndicalisation les salariés jugent indispensable le rôle des syndicats.

Alors pourquoi ça ne communique plus? Remarquons aussitôt que l'adhésion syndicale ne remplit pas seule les attendus du principe de contractualisation: d'autres " accommodements concernent aussi le type d'échange qui s'engage ». Depuis les progrès juridiques des institutions représentatives du personnel, «la contractualisation» syndicats/salariés peut s'accommoder de la participation aux élections professionnelles. De toute façon le syndicaliste est sur le lieu de travail et chacun peut lui parler même non syndiqué surtout si, en tant que délégué du personnel, celui-ci est l'élu de tous. Enfin le salarié remplit les exigences du principe de réciprocité dans la solidarité, s'il participe à des actions de masse telles que pétitions, manifestations ou grèves. L'adhésion n'a plus le monopole de la contractualisation de la communication entre syndicats et salariés. 

les enjeux ou de l'influence réciproque des éventuels interlocuteurs. Influence sur les enjeux? Parce qu'ils sont devenus des interlocuteurs officiels des divers pouvoirs, les syndicats sont contraints aux compromis aussi bien pour les solutions aux problèmes individuels que pour les revendications collectives. Ils sont donc suspects de « magouilles » et compromissions. Bien plus la mise en cause du principe d'influence sur les enjeux porte atteinte au principe de pertinence : les syndicats confédérés proposent des solidarités au sens le plus large: solidarités interprofessionnelle, interrégionale, inter-catégorielle, voir internationale, solidarité avec les exclus, les chômeurs, les consommateurs etc. L'organisation de telles solidarités suppose une vision globale de la société et de ses finalités, elle présuppose une «idéologie » et, à la limite, une certaine politisation du syndicalisme. Or l'échec de toute politique face à la crise de l'Ouest, et l'effondrement du marxisme à l'Est, réduisent le champ efficace de la solidarité espérée, à des solidarités de proximité catégorielles, professionnelles et localisées. Dès lors les syndicats confédérés qui refusent le corporatisme, sont réputés ne plus parler des « vrais » problèmes. D'où la non validation de la pertinence d'une interlocution avec eux. réciproques. D'où parle le syndicaliste ? S'il s'aligne toujours sur la position confédérale ${ }^{11}$ ou fédérale, il écoute mal sa «base » immédiate. S'il prend le parti de n'écouter que sa base adhérente ou électorale, à quoi bon qu'il soit confédéré ? Quant à l'influence du syndicaliste sur le salarié elle consiste moins à lui dénoncer des injustices sociales qu'à se justifier de ses positions dans les instances représentatives du personnel. Dès lors la belle réciprocité évoquée dans la situation potentiellement communicative (SPC) ne joue plus et le contrat de communication est rompu avant d'être engagé. syndicalisme : l'adhésion est le seul facteur de contractualisation de la communication avec un syndicat, sauf si le vote pour une instance représentative peut suffire à contractualiser cette communication; les enjeux et solidarités communs sont clairs tant que l'un des interlocuteurs ne devient pas un diplomate institutionnel aux multiples compromis ou l'otage d'une idéologie confédérale (donc institutionnelle) que l'Histoire petite ou grande a rendu utopique, sans compter les divisions que cela entraîne, et les difficiles justifications syndicales, alors que seul le patronat était réputé difficilement justifiable.

17 C'est le passage d'intralocuteur à interlocuteur qui perturbe le plus le contrat de communication. Ghiglione est efficacement précis quand il dit $^{12}$ que « ce qui constitue un individu en intra-locuteur ce sont les réponses qu'il a déjà obtenues et qu'il a constituées en système de savoir, de croyances, d'attitudes, de compétences", alors que "ce qui constitue un individu en interlocuteur potentiel ce sont les questions liées à un enjeu ».

Quelles sont les réponses que se sont appropriées les syndicalistes? L'histoire des luttes ouvrières certes, luttes sans lesquelles aucun progrès social ne se serait accompli du seul fait des divers patronats. Mais ils se sont appropriés aussi leurs nouveaux statuts conquis par ces luttes : celui d'interlocuteurs représentatifs, institutionnels et élus, des divers patronats privés et publics: délégués du personnel, comité d'entreprise, tribunal des prud'hommes, Conseil Economique et Social (national ou régional), organismes sociaux paritaires et une multitude de commissions mixtes officielles et légales. Le temps de travail syndical lui-même montre que le syndicaliste est plus l'interlocuteur des patrons ou de l'État que celui des salariés. Pire : cette interlocution syndicats/patronats peut, à la

Communication et organisation, 8 | 1995 
limite, fonctionner sans interlocution syndicats/salariés. C'est cette situation intralocutive que le syndicaliste assume à ses propres yeux et aux yeux des salariés dans sa tentative molle de s'adresser aux salariés. Dominique Labbé ${ }^{13}$ écrit : « Non seulement l'adhérent de base n'est pas recherché, mais il est souvent considéré comme un «poids mort » ou un « boulet ».

Quelle est la situation intralocutive du salarié "syndicable » : conformément à son idéal ou à son intérêt ou aux deux, il a appris sur le terrain qu'il a besoin de participer à des solidarités pour défendre ses intérêts moraux et matériels. La question qui le met en quête d'un contrat de communication est celle-ci : «quel est l'interlocuteur valable pour bénéficier de la solidarité ? «. Ce ne sont plus les syndicats mais les « institutions » créées grâce aux luttes syndicales: les organismes de protection sociale, les lieux où se dit le droit de travail (les divers tribunaux), le dialogue social direct avec ses dirigeants formés à rechercher un bon climat social et des employés motivés. Quant aux questions de la solidarité plus large, elles relèvent du jeu politique et non pas syndical. Et s'il faut réagir fort, nul besoin des syndicats, il y a les « coordinations » corporatistes ou non ${ }^{14}$. Dès lors les syndicats fonctionnent sur eux-mêmes dans la seule interlocution avec les patronats, sauf s'ils sont eux-mêmes gestionnaires directs de la protection sociale, comme dans les pays qui conservent, malgré la baisse, un fort taux de syndicalisation ; mais même là, les adhérents sont moins des syndiqués militants que des cotisants à un régime d'assurance mutualiste.

20 Le syndicaliste lui même fait figure de privilégié par son statut protégé et ses décharges, et non plus de combattant courageux, sauf bien sûr dans les PME où il est pourchassé comme au début du siècle. Il va dès lors faire du syndicalisme pour le syndicalisme dans une représentation du personnel qui n'est que l'expression de ses vues confédérales, et dans une expression à l'égard du patronat qu'il prend pour une représentation des salariés. C'est cette confusion circulaire de la communication-représentation et de la communication-expression que L. Sfez pour sa part appelle le tautisme ${ }^{15}$.

21 Cependant la situation resterait potentiellement communicative entre syndicats et salariés, si l'autre contrat de communication, celui des syndicats avec les patronats, était performant et performatif : mais ce n'est justement pas le cas.

\section{La rupture du contrat de communication entre les syndicats et les patronats publics ou privés}

De prime abord, la situation semble à nouveau communicative. Les chefs d'entreprises ou d'administrations ont besoin d'interlocuteurs sociaux constants et rompus au dialogue, ne serait-ce que pour éviter les coordinations où il est difficile de reconnaître juridiquement et à long terme un interlocuteur valable. Les syndicats (sauf à vouloir s'emparer du pouvoir économique ou politique, ce qui n'est plus à l'ordre du jour ou n'y a jamais été) ne peuvent rien obtenir s'ils ne l'obtiennent pas des directions. L'enjeu est d'autant plus clair qu'il ne semble ne dépendre que de ces deux interlocuteurs : équilibrer compétitivité économique de l'entité de travail et progrès social pour ses salariés et pour l'ensemble de la société civile. Les principes de pertinence, de réciprocité, de contractualisation paraissent donc aller de soi et convertir spontanément les intralocuteurs en interlocuteurs. D'ailleurs le contrat de communication prend la forme de la politique contractuelle que syndicats et patronats appellent de leurs vœux. Bien plus encore, la validation respective du principe de pertinence est devenue telle que la mort 
des idéologies entraîne l'avènement d'une même compétence discursive, d'un même usage de la raison, celui du « réalisme » économique tel qu'il semble s'imposer. Patronats et syndicats, orphelins du marxisme, ont un même référent : le réalisme économique des sciences économiques. Cela est très important : l'interlocution n'aura pas besoin grosso modo d'ajustement des référents et de leur pertinence. Du coup, si comme y insiste GHIGLIONE, "communiquer c'est co-construire une réalité à l'aide des systèmes de signes ", il n'y a plus qu'un seul système syntaxo-sémantique qui va produire une réalité avant même que les interlocuteurs ne la co-produisent : celui du "réalisme » économique de l'économie de marché. Par ailleurs, le principe d'influence, lui aussi nécessaire au contrat de communication selon Ghiglione, va se trouver ainsi complètement déséquilibré : dans le rapport compétitivité économique/progrès social, c'est d'abord le primat de la logique du réalisme économique, et de la compétitivité économique, car ils conditionnent le progrès social. Il ne faut pas mettre la charrue avant les bœufs. On comprend dès lors que les syndicats vont être soumis dans l'interlocution à l'initiative communicationnelle du pouvoir économique prioritaire. La revendication quoiqu'il en soit, risque d'être reléguée dans l'histoire syndicale.

Regardons bien l'usage à son avantage que le patronat va faire de son potentiel énorme d'influence discursive, linguistique, performative et pragmatique dans son contrat de communication avec les syndicats. Analysant ce qu'il appelle le tautisme de la décision et de la communication, Lucien Sfez prend un exemple ${ }^{16}$ : celui de la loi de 1990 sur l'autonomie des télécommunications. Dès 1985 on commence à en parler. 1987 : grève générale d'opposition au projet. 1988 « rapport Prevost » qui ne prétend que répertorier les demandes et soucis des divers acteurs. 1988-89 vaste consultation: 6 numéros d'un journal tiré à 500.000 exemplaires, 8000 réunions en interne pour le personnel, 6 colloques régionaux et 1 colloque national en externe. La contestation syndicale sera submergée.

La communication a sa logique propre, elle met en communication la multitude des partenaires, elle a le monopole de la problématique et des mots («France Télécom », « un avenir d'avance »), elle rend ainsi son contenu évident au bon sens, puisque le bon sens est justement ce qui est universellement communiqué. Il n'est pas possible de prendre la parole, ni même de penser, en dehors de cette novlangue, pour reprendre une formule d'Orwell.

Le contrat de communication entre syndicats et direction est rompu, mais ni par les uns ni par l'autre : au contraire, non seulement ceux-là mais tous les interlocuteurs sont validés. En revanche, il n'y a plus d'enjeux, car il n'y a pas de solution alternative. Ca parle, mais personne ne parle, le discours s'énonce de lui-même selon les règles de sa logique interne : c'est l'enfermement autistique et la tautologie de la communication, le "tautisme", quelle que soit la fiction de l'ouverture de la consultation (qui n'est pas le débat, mais l'universalisation du concept).

Ainsi chômage, inflation, salaires ressortent de logiques incontournables, où s'articule la communication de négociation. Encore faut-il un patrimoine commun de références pertinentes. C'est le réalisme auquel le salariat aussi se réfère. Mais si la «force des choses » l'emporte, à quoi bon s'engager dans l'action syndicale ? À la limite, il n'y a plus besoin d'interface syndicale patronat-salariat, puisqu'il n'y a pas d'alternative à la décision déjà prise. D’où la désyndicalisation. 
27 Les directions des ressources humaines lancent des idées, ne font pas de forcing, laissent mûrir et cheminer (campagne publicitaire aidant) pour les rendre évidentes, réalistes, pleines de bon sens. Elles utilisent le référent «entreprise » qui s'inscrit dans le champ sémantique de la référence "réaliste": en période de chômage, l'entreprise est l'instrument de survie de chacun. L'idée à faire passer emporte l'adhésion si elle est rationnelle c'est-à-dire conforme à la logique de sauvegarde de l'entreprise. Il s'agit même de lier l'idée nouvelle à une revendication sociale du personnel, non pas sous la forme traditionnelle du donnant-donnant, mais, quitte à la susciter chez le personnel, sous la forme d'une apparente amélioration sociale, que produira cette référence ellemême, laquelle n'a donc pas besoin d'autre compensation. Il en est ainsi du travail du dimanche, et de toute réforme de flexibilité négociée du temps de travail: c'est pour l'avenir de l'entreprise....et une meilleure répartition du temps libre du personnel. Les nouvelles directions communicantes " parlent » la communication sociale tout entière en l'énonçant elle-même par les deux bouts (direction et personnel), par les seules vertus d'une soi-disant logique incontournable. La neutralisation des syndicats est proportionnelle à la multiplicité des concertations, qui peu à peu accrédite le discours : le discours-expression devient discours-représentation d'une réalité incontournable. Voilà le tautisme. Le discours de la communication préexiste aux rencontres interlocutives et les informe, les met en forme. Ca ne marche pas toujours, mais ça marche de plus en plus souvent, du seul fait que le réalisme économique constitue le monopole de la compétence linguistique et discursive du principe de pertinence.

\section{Une éventuelle découverte : le tautisme comme neutralisation du contrat de communication sociale}

28 Le tautisme de Lucien SFEZ est un néologisme construit à partir de "autisme » et de «tautologie ${ }^{17}$ » : l'autisme signifie la fermeture solipsiste sur un monde préfabriqué et factice, et tautologie signifie dire la même chose en tournant en rond. Ici le tautisme est le fait d'un monopole du discours économique qui préfabrique une réalité, réalité prétendument co-exprimée par les interlocuteurs, mais qu'on retrouve intacte après leur interlocution jouée d'avance.

R. Ghiglione connaît le concept, même s'il n'utilise pas le mot tautisme. Citant la culture de BALI (page 102, opus cité), il met bien en évidence combien la structure de castes est exprimée par les trois sortes de langues de cette île et combien réciproquement les trois langues articulent la société de castes, à tel point que parler dans ces langues c'est d'emblée reproduire la structure sociétale, comme si celle-ci était une évidence.

30 À la page 206 l'Angolais et l'Angolaise conviés à s'exprimer dans leur langue sur la question de la dot vont par le mécanisme même de l'appareil linguistique et de l'ambiance illocutoire, reproduire dans le détail les schémas culturels que produit et qui produisent cette langue par son dit et son non-dit.

31 Dans ces deux exemples nous avons à faire à un tautisme où la communicationreprésentation et la communication-expression se confondent pour donner lieu à un système qui se reproduit et se confirme en et par lui-même. C'est ce que L. Sfez appelle «bande d'asservissement» ou « retournement de Feuerbach» pour qui «Dieu créé par l'homme s'impose comme son producteur ». 


\section{La pertinence du référent syntaxo-sémantique de l'économisme}

En ce qui nous concerne ici, le tautisme est celui de l'économie contemporaine où la représentation économique de la réalité n'est en fait que l'expression des hommes en interaction, et où cette expression s'aliène à cette fausse représentation du réel préfabriqué. Dans les situations potentiellement communicatives entre Etat, patronats, syndicats et salariés, l'enjeu interlocutif est l'avenir économique et social, et le référent intralocutif est le présent économique et social. On pourrait croire que le passage du présent au futur sera le résultat de l'interlocution des partenaires sociaux. En fait c'est la discursivité même du discours commun qui dicte ce qui doit être pensé, dit et fait selon la "pensée unique ", celle de l'économisme consensuel. L'économisme présente l'économie comme un déterminisme inexorable qui n'obéirait pas à la volonté des interlocuteurs économiques et sociaux, mais à une logique qui s'autorégulerait selon ses propres lois : malheur à ceux qui tenteraient de désobéir à cette irrécusable " réalité » que disent les instituts et les experts, comme les tribunaux disent le droit et comme les savants disent la vérité. $B$. Walliser n'hésite pas à parler de «règles de fonctionnement déterministes ${ }^{18}$ ». Simon en 1945 écrit déjà que "la décision n'était plus un acte libre, mais le fruit d'un processus prédéterminé », et en 1965 il ne voit finalement guère plus de marges de manœuvres, sinon sur les microchoix des moyens qui, de fil en aiguille, déterminent les fins ${ }^{19}$.

Même si nombre de grands économistes s'en défendent (René Passet, Maurice Allais, etc.), ainsi que beaucoup de spécialistes moins connus, les sciences économiques des grands instituts médiatiques (INSEE, OCDE, etc..) ne sont plus seulement cognitives et descriptives, leur discours est d'emblée décisionnel, normatif, performatif. S'élaborent ainsi des modèles économiques de politique gouvernementale tel que celui de Lucas (1972), et son correctif de Barro-Gordon (1983). Presque tous les interlocuteurs de la politique contractuelle ont assimilé les règles d'or de la désinflation compétitive, du monétarisme et de la priorité de la lutte contre l'inflation avant celle contre le chômage, quitte à aggraver le chômage dans un premier temps. Il y aurait des lois économiques comme il y a des lois physiques (cf. Alfred Sauvy Y), et le libéralisme apparemment si peu enclin à se soumettre à des lois dirigistes, n'arrête pas de décrypter et de suivre les lois imprescriptibles de «la main invisible» de A. Smith. Quant à ses anciens détracteurs, ils ont remplacé feus Marx et Lénine par l'INSEE et l'OCDE, faute de pères et de repères plus fiables. Dans la crise des finalités, on se replie sur les seules lois du fonctionnement. R. Passet écrit: "le règne de l'économisme comme idéologie, avouée ou non, et critère suprême des orientations du développement, débouche aujourd'hui sur la question des finalités du système productif et des valeurs que ce dernier devra servir ${ }^{20} »$. En attendant, c'est le tautisme de l'économie au service de l'économie. Du référent aux enjeux, il n'y a plus de place pour l'interlocution, il n'y a plus d'enjeux mais seulement un référent qui projette un futur selon ses propres lois syntaxo-sémantiques.

L'interlocution a lieu mais elle est devenue neutre quant à ses résultats obligés.

Le référent sans références et la langue de bois des contrats de communication sociale.

Le consensus économiste intervient tant au niveau des principes du contrat de communication (principes de pertinence de la même compétence linguistique et discursive au départ) qu'à celui des règles, règles de la discursivité qui joue tout au long de l'interlocution sociale. L e principe de pertinence n'est pas le seul concerné. Le 
principe même de contractualisation (toujours de GHIGLIONE) n'est-il pas garanti par l'économie elle-même? Regardons comment s'opère l'identification de la contractualisation économique et sociale et de la contractualisation communicative.

Lévi-Straus ${ }^{21}$ écrit à ce sujet que " dans toute société, la communication s'opère au moins à trois niveaux: communication des femmes, communication des biens et services, communication des messages ». Seule cette dernière semble concerner le concept de communication de Ghiglione. Ce serait sans compter sur l'économie qui comme toute science est d'abord un système symbolique avant de représenter une "réalité ». LéviStrauss situe bien l'avènement de l'économie quand il dit: « Bien que les biens et services ne soient intégralement ni des symboles, ni des signes, on a besoin de symboles et de signes pour échanger dès que le système économique atteint un certain degré de complexité ». Dès lors l'auteur de l'Anthropologie Structurale peut évoquer la conjonction de la science économique et de la science linguistique «pour fonder une discipline commune qui sera la science de la communication ».

38 L'économie dit symboliquement la communication en actes (échanges de biens et services) ; la science de la communication dit la communication en verbe par symboles. La communication symbolique des messages sociaux va s'identifier à la communication symbolique des échanges économiques (l'Economie) du seul fait que l'économie actualise «de force» le contrat de communication entre les acteurs économiques et sociaux aux prises avec leurs échanges mutuels.

39 Parce que l'Economie communique des symboles pour communiquer des biens et des services vitaux, elle est l'ordre symbolique moderne qui s'impose à tout langage social, une sorte de métalangage obligé de la communication sociale. Les interlocuteurs pourront-t-ils être co-sujets d'une énonciation d'ores et déjà structurée par la science économique? En fait pour parodier Saussure parlant de la langue, nous dirons que « l'économisme est un système qui ne connaît que son ordre propre » et ne laisse que peu de marge de liberté aux interlocuteurs sociaux, enfermés dans un contrat de communication scellé dans la « réalité » avant qu'ils ne parlent.

Mais est-ce bien la réalité ? Le référent est il fiable? En fait chaque symbole du système syntaxo sémantique de la communication économique et sociale ne renvoie pas à une réalité extérieure à ce système symbolique (les choses échangées), mais ne renvoie qu'à d'autres signifiants internes au même système. La monnaie par exemple est un signifiant dont le signifié est une valeur d'échange. Mais comme dans la linguistique saussurienne d'ailleurs évoquée par Ghiglione ${ }^{22}$, le rapport signifiant/signifié est arbitraire, et la valeur d'échange de la monnaie est fluctuante, non en raison d'un changement de la richesse réelle désignée par cette monnaie (le PIB par exemple) mais en fonction des fluctuations d'autres signifiants que sont les autres monnaies. Soutenir une monnaie est moins maintenir la richesse économique globale qu'elle est censée représenter (rapport signifiant/signifié) que de faire acheter cette monnaie par d'autres monnaies (rapport signifiant/signifiant) pour en faire monter le cours. Une monnaie ne s'évalue plus en valeur d'échange de biens et services, mais en autres monnaies : 1 franc vaut davantage $X$ mark que telle quantité de pain. Augmenter les taux d'intérêt n'est pas augmenter le pouvoir d'achat d'une monnaie (référent réel) mais son pouvoir d'être vendue elle-même chère. La spéculation ne joue que sur les signifiants sans signifiés ni référent chosal. Le monétarisme n'est qu'un culte rendu à des référents eux-mêmes sans référence extérieure aux systèmes monétaires. Peu importe qu'il n'y ait plus de sens du tout comme tous ces pétrodollars qui circulent à travers le monde en tant qu'équivalents universels, 
mais qui ne valent rien faute d'être convertibles par la Banque centrale des USA. Il en va de même des déficits budgétaires ou commerciaux qui, à l'instar du crédit excessif, introduisent des signifiants sans signifiés; personne ne conteste quand ces signifiants sont en eux-mêmes des équivalents universels auto-références, tels que le dollar. Et quand le crédit est appliqué aux achats ou ventes monétaires spéculatifs, on aboutit au signifié 0 de la bulle financière. La dette du Tiers-Monde n'est dans les banques qu'un jeu d'écriture officiel (un signifiant) sans référence, puisque insolvable, mais la nonreconnaissance de ce non-lieu par le Tiers-Monde entraînerait la faillite des grandes banques où la dette figure aussi dans les actifs. C'est pourquoi Lévi-Strauss a raison, du moins dans notre exemple économiste, de dire que les «symboles sont plus réels que ce qu'ils symbolisent », et il aurait pu dire ce que LACAN écrit par ailleurs "c'est le monde des mots qui crée le monde des choses ${ }^{23} »$.

41 Alors communiquer, comme le dit Ghiglione, c'est bien " co-construire une réalité à l'aide des systèmes de signes", sauf que, s'agissant de la communication des acteurs économiques et sociaux, le système de signes pré-existe à l'interlocution, où les interlocuteurs sont prisonniers du système de signifiants flottants et de ses nonréférences. L'autisme et la tautologie règnent en maître : c'est le tautisme.

42 N'est-on pas en présence d'une langue de bois qui faute d'ancrage dans la réalité ne produit qu'une réalité truquée sur la fausse pertinence consensuelle de laquelle s'établit le contrat de communication sociale?

43 À la page $217^{24}$, Ghiglione rend compte de l'exemple de ce psychiatre qui veut faire le point avec un schizophrène, lequel s'y refuse en fuyant dans un discours autistique : «Bon alors ce qui... », « coffélec » interrompt le malade. "C'est quoi coffélec? ». « c'est ratatouille moderne... ça qui donne la chiasse». Ghiglione conclut que ce dialogue témoigne moins d'un désordre de la pensée que d'un refus de s'engager dans le contrat de communication proposé par le médecin. Toute proportion gardée, c'est un peu la fausse situation potentiellement communicative du dialogue social. Soit le syndicaliste (rarement depuis 20 ans de crise) refuse radicalement la pertinence du discours économiste, et alors en quelque sorte, pour l'opinion publique, l'État et le patronat il répond « coffélec »; soit tous les acteurs jouent le jeu de cette économisme et c'est encore " coffélec », une fuite et une peur collectives devant la fatale fragilité de l'économie locale et mondiale, mais cette fois ci un " coffélec » où chacun se comprend en participant au même délire - refuge d'une langue de bois prétendument réaliste. Grande rigueur discursive pour une logique qui ne tourne que sur elle même dans l'autisme et la tautologie. Mais le contrat de communication ne fonctionne pas, il se contente d'accompagner vaguement l'attentisme de la sortie du tunnel, qui ne peut advenir que par la logique interne de l'économisme. Cependant on fait semblant de le faire fonctionner.

Paradoxes, intralocuteurs et interlocuteurs du contrat de communication sociale.

Le contrat de communication Patronats-Etat/Syndicats se transforme ainsi en simulation et en jeu de rôles, car les règles (selon le concept de Ghiglione) sont "pipées » dès le départ. Les règles situationnelles instituent les partenaires dans leurs statuts de coresponsables de l'avenir économique et social. Mais les règles discursives, celles qui régissent pendant l'interlocution les structures linguistiques, métaphoriques et rationnelles des énonciations et compréhensions réciproques, sont celles de l'économisme, selon lequel aucun des interlocuteurs n'a prise vraiment sur l'économie et l'avenir : il n'y a plus de pilote dans les entreprises et les États. C'est ce paradoxe entre les 
règles situationnelles et les règles discursives, (chacune d'entre elles sincèrement assumées) qui crée la simulation, laquelle fait du contrat de communication une fiction de jeux de rôles. On joue à faire semblant d'être co-responsable potentiel, mais on abdique devant l'expertise économiste. Ne sommes nous pas ici dans une situation proche de l'exemple de ce que sont les règles du contrat de communication pour Ghiglione ${ }^{25}$ : celui de la publicité ? «La femme est une île, Fidji est son parfum ». Règle situationnelle qui instaure le publiciste et les consommateurs dans un contrat de communication accepté par chacun. Règle discursive « qui institue le destinataire en agent d'une quête (le rêve et non le produit) » dont le produit n'est qu'un moyen magique d'accès. Cette discursivité ne fait-elle pas de l'acheteur potentiel une dupe, mais qui sait qu'il est dupe ? Dans le contrat de communication sociale, le syndicaliste connaît sa duplicité: il va jouer le rôle revendicatif attendu, mais dans les limites étroites de l'économisme consensuel.

B. Walliser (déjà cité) parle de conditions de coordinations qui postulent la même rationalité cognitive chez tous les acteurs, et J.F. Amadieu ${ }^{26}$ n'hésite pas à dire qu' ' il pose le postulat que les employeurs et les salariés sont mus par une même rationalité économique ", tel l'homo œconomicus. Chacun peut anticiper le rôle de l'autre car l'interlocution est pré-programmée par la discursivité socio-économique consensuelle. Chacun parle selon son rôle et tout le monde ne dit pas la même chose, mais tout le monde est "parlé " par là même langue économiste (sauf quelques rares déviants). Non seulement les interlocuteurs ne sont plus vraiment sujets d'énonciation de leur dialogue, ni co-producteurs d'une réalité, mais ils font partie de l'énoncé du discours économiste qui les ordonne dans son système : un blocage des salaires doit faire réagir les syndicats, cette réaction est prise en compte dans une rationalité économétrique qui l'intègre a priori dans les limites consensuelles du possible rationnel.

Les intralocuteurs sont devenus mondialistes: à la question "d'où parles-tu?», le syndicaliste se répond à lui-même qu'il parle au nom des intérêts moraux et matériels des salariés, mais il se dit aussi que l'économie ne s'articule désormais qu'au niveau mondial, niveau sur lequel personne n'a prise : il faut se contenter de jouer le jeu de cette logique anonyme de la "main invisible » de l'économie du marché planétaire. Sorte de retour au fatum romain et perte de la maîtrise du destin de l'humanitée ${ }^{27}$. D'autant plus que nous avons déjà dit (Chap. II) que la représentati.vité syndicale est faible, tout comme celle du patronat (tout aussi mondialiste dans ses références). L'économisme et le mondialisme font partie de l'appropriation culturelle des intralocuteurs de la communication sociale.

Patrons et salariés (mais pas les syndicats) sont paradoxalement devenus très individualistes alors que le consensus est à son comble. Cet individualisme est pourtant la conséquence directe de la culture économiste mondiale: Si collectivement, et interlocutivement, on ne peut rien y faire, c'est le « sauve-qui-peut » et le «chacun pour soi »: d'où la désyndicalisation aussi bien salariale que patronale (quelle est la représentativité réelle du CNPF ?).

Tous les interlocuteurs de la communication sociale sont soumis au paradoxe d'un double bind, que Ghiglione utilise beaucoup par son emprunt limité à l'école de Palo Alto ${ }^{28}$. Ghiglione reprend l'exemple classique de la mère schizophrénisante qui s'adresse à son fils psychotique: «j'espère que tu ne feras pas ce que je te demande». Il appelle cela "contradiction entre la signification syntaxo-sémantique et la signification des indicateurs d'énonciation" (l'attitude non dite de ne pas embrasser de la mère, par exemple). Or nous avons la même fausse situation potentiellement communicative entre Patronats et syndicats. Les syndicats font preuve d'une gesticulation illocutoire agressive 
du genre: «l'automne sera chaud». C'est leur côté intralocutionnaire. Mais dans l'interlocution avec les patronats, ils sont prisonniers de la rationalité syntaxosémantique consensuelle. Il s'agit aussi dans l'interlocution de ne pas perdre la face ${ }^{29}$, compte tenu de la gesticulation médiatique. Dédoublement de la personnalité du syndicaliste ("schizophrénie ») ajouté à cet enjeu devenu prioritaire (" ne pas perdre la face») peut conduire à la «schizophrénie paranoïaque » du syndicaliste. C'est du moins celui qui sait le mieux la simuler ou l'assumer qui occupera le devant de la scène. Il n'y a pas d'autre choix, car les salariés, eux-mêmes créent ce double bind: en tant que salarié de tel établissement je veux ici et maintenant les avantages sociaux quels que soient les impératifs économiques ${ }^{30}$ (vieux réflexe de contrat de communication corporatiste), mais en tant que citoyen je suis peu ou prou acquis au consensus économiste. Dès lors le syndicalisme a toujours tort: coopératif il est traité de collaborationniste inefficace, déviant il est considéré comme irréaliste et dangereux par les salariés eux-mêmes. C'est ainsi que la schizophrénie part des individus qui par exemple ont «tendance à la dissociation croissante entre vote politique et appartenance syndicale ${ }^{31}$ ». La CGT se demande ainsi « s'il existe le risque d'une CGT hésitante et menacée d'accompagner ou de cogérer les politiques de crises $^{32}$ ».

Bref le tautisme économiste s'est emparé du monopole du discours et des actes. Tous les gouvernements ne font-ils pas à peu près la même politique? Les contrats de communication ont gardé du passé l'apparence contestataire illocutoire (les indicateurs d'énonciation), mais sur le fond leur interlocution est lénifiante et attestataire du terrorisme intellectuel de l'économisme-roi (ne serait-ce que sous la pression de l'opinion publique).

51 Ne disons pas que les syndicats n'ont pas su s'adapter : ils sont soumis au double bind de divers paradoxes de leurs contrats de communication, tant avec les salariés, qu'avec les patronats. Tributaires du climat d'opinion déçu des idéologies et replié sur le pseudoréalisme économique, ils sont enfermés dans le tautisme économiste mondialiste, tout comme les grands médias.

Certes les syndicats ont eu tort de s'institutionnaliser, c'est la rançon de leur victoire sociale et la cause principale de ruptures du contrat de communication avec les salariés. C'est aussi le moyen de leur intégration dans les rético-rationalités économiste et mondialiste qui neutralisent le contrat de communication avec les patronats et l'État. Syndicalisme trop confédéral et pas assez de terrain ici et maintenant ${ }^{33}$.

53 Toujours est-il que l'apport de l'appareil conceptuel de Ghiglione, et dans une moindre mesure ici celui de Sfez, ont été éclairants et synthétisants d'une complexité.

54 La relance économique en atténuant la crise du chômage et en donnant « du grain à moudre " permettra à coup sûr une redynamisation des contrats de communication sociale et donc de la syndicalisation. Le moment peut être ne tardera pas. Mais on ne reviendra pas en arrière.

La quadrature du cercle passe par un syndicalisme de mise en perspective sur le terrain de la logique économiste et de la logique de la dignité de l'homme. Contester l'économisme sans rompre les contrats de communication avec les partenaires économiques et sociaux. I.G. Mettal y a parfois réussi en Allemagne.

La logique économiste, comme toute logique, n'est logique que par rapport à ses postulats et pour une complexité changeante. Il faudra donc provoquer des situations potentiellement communicatives dont l'enjeu sera la méta-communication sociale elle- 
même: quelle société? Quelles finalités? Quels postulats pour une nouvelle logique économique? L'échelon n'est plus ici le terrain mais celui des confédérations mondiales ou au moins européennes. Encore faut-il sur le terrain comme au sommet, se libérer de la « pensée unique » et du trop peu de représentativité. La seule clarté est le maintien intact du capital intralocutif et des compétences interlocutives des syndicalistes. Mais quels seront les Hume qui réveilleront les Kant d'aujourd'hui de « leur sommeil dogmatique »?

\section{NOTES}

1. Cf. L'homme communicant de Rodolphe Ghiglione (Éd. Armand Colin) Exemple de l'homme et de la femme angolais parlant de la dot (p. 206 et sq.) ou exemple des 2 universitaires de gauche (Français et Italien) parlant de Berlinguer (p. 213 et sq.)

2. Opus cité : publicité des parfums Fidji p.5. Dictionnaire critique de la communication (PUF). Article de R. Ghiglione p. 461 sur la publicité du Crédit Lyonnais.

3. CERAT-IEP Grenoble.

4. Nombre de syndiqués par rapport à la population active salariée

5. Mouvement social (Ed. Ouvrières) n 162 mars 1993

6. Colloque international de l'Institut National du Travail à Lyon les 4 et 5 juin 1992 (publié en 1993 par l'IMT).

7. Voir déclaration de J.F Amadieu lors du $1^{\text {er }}$ Congrès régional des relations industrielles des Amériques (Québec - Août 1988).

8. Le peuple (organe de la CGT) juillet 1993

9. Opus cité note

10. Voir par exemple La question syndicale (Calman-Lévy) de P. Rosan Vallon.

11. Les médias donnent une identité collective à chaque centrale, à chaque sigle ; et la communication syndicale a besoin de cette médiatisation

12. Dictionnaire critique de la communication (PUF), article de R Ghiglione p. 460.

13. Revue de L'IRES n 16, p. 92.

14. La manifestation pour l'école privée (1984) ou pour l'école publique (1994) n'ont pas été corporatistes et ont débordé largement les capacités de mobilisation syndicale (ou même celles des partis).

15. Concept important de Lucien Stez que l'on retrouve tout aussi bien dans La critique de la communication (Le seuil 1988), La communication (Que sais-je ? 1994), Dictionnaire critique de la communication (PUF 1993).

16. Que notre expérience syndicale contemporaine de l'exemple peut attester.

17. Cf. note 15 .

18. Instrumental rationality and cognitive rationality in « Theory and decision » 1989.

19. Administrative Behaviour, 1965, Éd. Des Organisations en collaboration avec March ».

20. Il serait intéressant de comparer cet asservissement à l'économisme à l'arraisonnement (das Gestell) de l'homme par la technique selon Heidegger. Ce dernier n'est pas loin de dénoncer « l'économisme » quand l'homme de la Forêt Noire écrit : « Le garde forestier qui mesure le bois abattu et qui, en apparence, suit les mêmes chemins et de la même manière que le faisait son grand-père, est aujourd'hui, qu'il le sache ou non, commis par l'industrie du bois » (La question de la technique. Gallimard). 
21. Anthropologie structurale, Plan, 1958, p. 79 et 329.

22. Opus cité à la note 1, p. 39.

23. À l'inverse de ce que dit Marx, on pourrait avancer que les superstructures (symboliques) conditionnent les infrastructures économiques et sociales.

24. Opus cité à la note 1 .

25. Opus cité à la note $1:$ p. 103-104.

26. Cf. note 7, et nombreux autres conférences et articles.

27. On « attend », par exemple, la relance américaine.

28. Opus cité à la note $1:$ p. 229 par exemple

29. Rodolphe Ghiglione y accorde beaucoup d'importance. Cf. opus cité en note 1, p. 213

30. Dominique Labbé (déjà cité) sourient, contre beaucoup d'observateurs que « le syndicalisme à la française » était un syndicalisme de « terrain » corporatiste et catégoriel.

31. J. Freyssinet, Le mouvement social, p. 10, n 162, janvier-mars 1993.

32. Le peuple (organe de la CGT) juillet 1993.

33. Simple indication symptomatique : en 1970, la CFDT comptait une trentaine de permanents confédéraux et la CGT une centaine. Aujourd'hui où la désyndicalisation est patente, ces chiffres ont au moins quintuplé et ont donné lieu à des fourmilières d'experts

\section{RÉSUMÉS}

Si la désyndicalisation est la désaffection des salariés à l'égard des confédérations syndicales, n'est ce pas dû au fait que syndicats et salariés n'ont plus grand chose à se dire ou qu'ils ne se comprennent plus, pourquoi dès lors ne pas analyser le phénomène en termes de ruptures de " contrats de communication", d'autant que les sociologues se perdent en conjectures? Les sciences de la communication peuvent peut-être apporter une synthèse là où l'étiologie de la désyndicalisation s'éparpille. Mais quelle synthèse? Notre découverte serait celle d'un «tautisme " communicationnel selon le mot de Lucien SFEZ, principalement selon nous, d'un «tautisme » économiste, c'est-à-dire d'un système symbolique économique qui s'autorégule selon son ordre propre.

If declining union membership is due to workers'disillusion with them, isn't this due to the fact that unions and workers no longer have anything to say to each other or that they don't understand each other? why not analyse this phenomenon immediately in terms of breaking " communication contracts", especially as sociologists are lost in conjectures ? Communication sciences might be able to synthesise just where the etiology of union decline is incoherent. But what kind of synthesis? We have discovered a communication «tautisme " according to Lucien Sfez, which is mainly, according to us, an economic "tautisme" that is to say a symbolic economic system which is self regulating according to its own order. 


\section{AUTEUR}

BERNARD IBAL

Docteur d'État en philosophie. Vice-Président confédéral de la CFTC. Consultant d'entreprise et ancien président de L'IRES (Institut de Recherches Économiques et Sociales) 